Las experiencias, también centradas en el tema de la cultura y la educación en el ámbito de las tecnologías multimedia, giran en torno a temas como las nuevas tecnologías en documentación e información aplicadas al estudio del patrimonio cultural, el acceso a través de Internet a museos, bibliotecas $y$, en general, a las instituciones relacionadas con el patrimonio, etc.

A continuación, siempre volviendo al menú principal, podemos utilizar el ratón para obtener información sobre las mesas redondas que se desarrollaron a lo largo del encuentro. En éstas se pueden encontrar propuestas acerca de la gestión creativa de actividades que tengan por objeto la difusión del patrimonio cultural en la sociedad de la información y de proyectos que aborden la presencia de museos e instituciones culturales en el ámbito multimedia.

Asimismo, se hace referencia a los principales desafíos de la era digital en relación al patrimonio cultural: el libre acceso a las imágenes y la cultura por parte de los ciudadanos y la introducción de las nuevas tecnologías digitales en los diferentes sistemas educativos.

Por otro lado, el CD-ROM también presta atención a las visitas organizadas que se lleva-

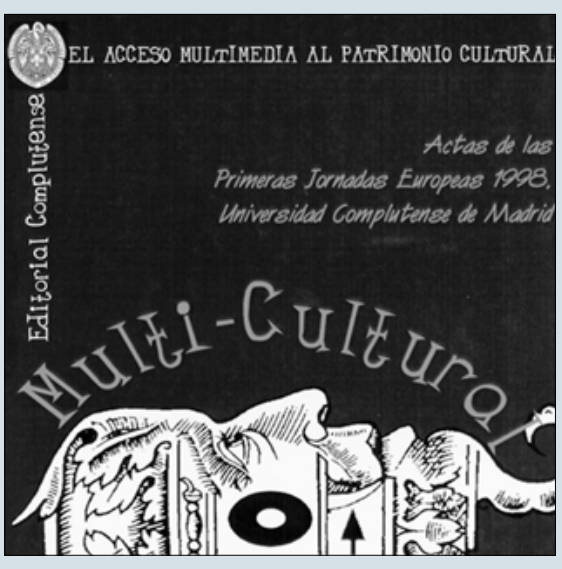

ron a cabo durante la celebración de las Jornadas al Museo Nacional Centro de Arte Reina Sofía, al Museo Interactivo del Libro y al Museo Internacional de Electrografía de Cuenca. En algunos casos, el CD permite realizar un recorrido virtual por estas instituciones a través de imágenes tanto de las dependencias como de sus fondos.

En último lugar, encontramos un apartado dedicado a las conclusiones del encuentro. En ellas se destaca la nueva concepción, más amplia que la tradicional, del patrimonio cultural, de su valor de uso irremplazable y de la necesidad de integrar este patrimonio en la vida cotidiana.

Esta nueva concepción puede encontrar en la tecnología hipermedia una vía de desarrollo de alcance insospechado, que permitirá difundir una visión amplia, científica y amena de la cultura, aprovechando el abanico de posibilidades que ofrece la interactividad de acercar al usuario a la comprensión y el disfrute del patrimonio.

\section{Mención de responsabilidad}

Dirección: Arturo Colorado Castellary

Dpto. de Comunicación Audiovisual y Publicidad II Universidad Complutense. Madrid

Diseño gráfico, fotografias y programación en lingo: Eva $M^{a}$ de la Torre Ruiz

Edición: Editorial Complutense

I.S.B.N. 84-89784-90-8

\section{Requerimientos técnicos recomendados}

- Windows 95

- Pentium 133 Mhz

- RAM 24 Mb

- Display Millones de colores (24 bits)

- CD-ROM 20x

- Tarjeta de sonido

María Victoria Madrid Díaz Centro de Documentación, IAPH

\title{
Información Bibliográfica sobre Tesauros de Patrimonio Histórico
}

En la selección bibliográfica que presentamos se recogen aquellos Tesauros, publicaciones y herramientas afines que por su contenido, se encuentran relacionados de forma global o parcial, con algunas de las disciplinas que integran el Patrimonio Histórico. En algunos casos su temática induce a pensar, a simple vista, que su relación con el Patrimonio Histórico es nulo, pero al introducirnos en el corpus interno encontramos una serie de desciptores relativos a distintas parcelas del conocimiento que se pueden aplicar al Patrimonio Histórico. Esta selección la acompañamos de monografías y artículos que tratan sobre el Tesauro de Patrimonio Histórico Andaluz, bibliografía no muy extensa pero que muestra el pasado, presente y futuro del lenguaje documental del Sistema de Información del Patrimonio Histórico Andaluz (SIPHA).
ART and Architecture Thesaurus. New York: Oxford University Press, 1994. (Published on behalf of the Getty Art History Information Program).

ASIS thesaurus of information science and librarianship. Medford (New Jersey): Published for the American Society for Information Science, 1994.

The BRITISH Museum materials thesaurus. Cambridge: MDA in association with the British Museum, 1997.

BULLETIN of the vocabulary program. Los Angeles: Getty Information Institute, c.1997. Semiannual. N N I (1997)-

CENTRO de Estudios y Experimentación de Obras Públicas. Tesauro de puertos y costas. [Madrid]: CINDOC, 1996.
DOMENECH FERNÁNDEZ, Silvia. Tesauro BIMA. Barcelona: Archivo Municipal, 1997.

FERREZ, Helena Dodd; BIANCHINI, Maria Helena $S$. Thesaurus para acervos museológicos. Rio de Janeiro: Fundaçao Nacional Pró-Memória, 1987. (Série Técnica, I)

FINDEX: facet-oriented indexing system for architecture and construction engineering... Sttutgart: Irb-Verlag, 1985.

GARNIER, François. Thesaures iconographique: système descriptif des représentations. Paris: Le Léopard d'or, D.L. 1984.

GIL LABLANCA, Rafael. Tesauro de Obras Hidraúlicas. Madrid: CINDOC, 1996. 
GROUPE d 'Antropologie historique de l'occident medieval. Thesaurus des images medievales: pour la constitution des bases de donnes iconographiques. Paris : École des Hautes Etudes en sciences sociales, 1993.

GRUPO de Archiveros Municipales de Madrid. Materiales para un Tesauro de Archivos Municipales. Madrid: Comunidad de Madrid, 1999.

GUIDE to indexing and cataloging with the art \& architecture thesaurus New York : Oxford University Press, 1994. (Published on behalf of the Getty Art History Information Program)

HOLM, Stuart A. Guidelines for constructing a museum object name thesaurus. Cambridge: Museum Documentation Association, 1993.

LAVELL, Cherry. British archaeological thesaurus: for use with British archaeological abstracts and other publications in British archaeology. London: Council for British Archaeology, 1989.

LIBRARY of Congress. Prints and Photographs Division Thesaurus for Graphic Materials. Washington D.C. : Cataloguing Distribution Service, Library of Congres, 1995.

MULTILINGUAL glossary for art librarians: English with indexes in Dutch, French, German, Italian, Spanish and Swedish. Edited by IFLA Section of Art Libraries. Munchen, New Providence : K.G. Saur, 1996.

PETERSEN, Toni. Directory of thesauri for object names. Williamstown, ICOM-CIDOC, 1994. (Plurilingual: english, french).

RIZZO, Stefano. Bibliografia internazionale di thesauri = International bibliography of thesauri. Roma : Camera dei deputati, 1987.

ROZO RíOS, Dora Susana. Tesauro en documentación e información. Bogotá: Instituto Colombiano para el Fomento de la Educación Superior, 1980.

RUBIO LINIERS, María Cruz. Tesauro de Historia Contemporánea de España. Madrid: CINDOC, 1999.

SANTOS CANALEjO, Elisa Carolina de. Tesauro Archivo. Madrid: Ministerio de Asuntos Exteriores, 1994.
STRATEN, Roelof van. Iconography, indexing, ICONOCLASS : a handbook. Leiden, The Netherlands : Foleor, 1994.

TERMINOLOGY for museums. International Conference of Museum Documentation Association (2.1988.Cambridge). Cambridge: The Museum Documentation Association, 1988.

TESAURO de Defensa. [Madrid]: Ministerio de Defensa. Subdirección General de Publicaciones, 1996.

TESAURO de Medio Ambiente. Madrid: Ministerio de Obras Públicas y Urbanismo, 1990.

TESAURO de Patrimonio Histórico Andaluz (versión 0). Granada: Instituto Andaluz del Patrimonio Histórico, Comares, 1998.

TESAURO ISOC de Urbanismo. Madrid: Consejo Superior de Investigaciones Científicas, 1992.

TESAURO de la UNESCO: lista estructurada de descriptores para la indización y la recuperación bibliográficas en las esferas de la educación, la ciencia, las ciencias sociales y humanas, la cultura, la comunicación y la información. Paris: UNESCO, 1995. (Plurilingual: english, french, spanish).

TESAURUS de Urbanismo. Madrid: Ministerio de Obras Públicas y Urbanismo, 1983.

THESAURI for museum documentation: the proceedings of a workshop held at the Science Museum, London. (Museum Documentation Association Terminology Working Group. Workshop. London. 1992). Cambridge: Museum Documentation Association, 1992. (MDA occasional paper, 18)

THESAURUS of archaeological site types. London: Royal Commission on the Historical Monuments of England and English Heritage, 1992.

THESAURUS de Información y Comunicación. París, La Haya: UNESCO/FID, 199I.

THESAURUS of monument types : a standard for use in archeological and architectural records. Swidon : Royal Commission on the Historical Monuments, National Monuments Record Centre, 1995.
THESAURUS for museology: based of the ICOM-ICOMOS Cultural Heritage Thesaurus. London: ICOM, 1990.

USER friendly : a newsletter for AAT users. Williamstown, MA: Art and Architecture Thesaurus, 1993. Quaterly. Vol. I, nI (1993)-

\section{Referencias bibliográficas sobre el Tesauro de Patrimonio Histórico Andaluz:}

BAENA PADILLA, E, CANCA CUENCA, J, MARTÍN PRADAS, Antonio. El programa de gestión del Tesauro de Patrimonio Histórico Andaluz. Boletín PH, n²4, 1998, pp. I I0-I I6.

GARCÍA GutIÉRREZ, Antonio. Principios de lenguaje epistemográfico: la representación del conocimiento sobre patrimonio histórico andaluz. Granada: Instituto Andaluz del Patrimonio Histórico, 1998.

MUÑOZ CRUZ, Valle. La normalización del Sistema de Información del Patrimonio Histórico de Andalucía: el Tesauro de Patrimonio Histórico. Boletín PH, n 14, 1996, pp. I I0-I 15.

MARTíN PRADAS, Antonio. El lenguaje documental del sistema de información del Patrimonio Histórico Andaluz (SIPHA): el tesauro del Patrimonio Histórico Andaluz. En I Jornadas Andaluzas de Documentación (Jadoc'97). Sevilla: Asociación Andaluza de Documentalistas, 1997. Pp. 275-280.

MARTíN PRADAS, Antonio. El Tesauro de Patrimonio Histórico Andaluz. En Actas II Jornadas de Museología: El Museo Centro de Documentación. Revista de la Asociación Profesional de Museólogos de España, $n^{\circ} 2$. Madrid 1997, pp. 260-267.

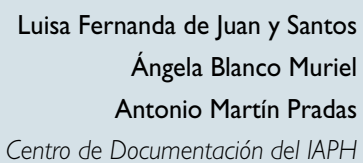

Centro de Documentación del IAPH 